\title{
High-resolution combined arterial spin labeling MR for identifying cerebral arterial stenosis induced by moyamoya disease or atherosclerosis
}

\author{
Jingyuan $\mathrm{Ya}^{1,2,3}$, Da Zhou ${ }^{1,2,3}$, Jiayue Ding ${ }^{1,2,3}$, Yuchuan Ding ${ }^{3,4}$, Xunming Ji ${ }^{2,3,5}$, Qi Yang ${ }^{2,3,6}$, Ran Meng Me,3 $^{1,2,3}$ \\ ${ }^{1}$ Department of Neurology, Xuanwu Hospital, Capital Medical University, Beijing 100053, China; ${ }^{2}$ Center of Stroke, Beijing Institute for Brain \\ Disorders, Beijing 100069, China; ${ }^{3}$ Department of China-America Institute of Neuroscience, Xuanwu Hospital, Capital Medical University, Beijing \\ 100053, China; ${ }^{4}$ Department of Neurosurgery, Wayne State University School of Medicine, Detroit, Michigan, USA; ${ }^{5}$ Department of Neurosurgery, \\ ${ }^{6}$ Department of Neuroimaging, Xuanwu Hospital, Capital Medical University, Beijing 100053, China \\ Contributions: (I) Conception and design: J Ya, R Meng; (II) Administrative support: Y Ding, X Ji, Q Yang, R Meng; (III) Provision of study materials \\ or patients: J Ya, D Zhou, J Ding; (IV) Collection and assembly of data: J Ya, D Zhou, J Ding; (V) Data analysis and interpretation: J Ya, D Zhou, J \\ Ding; (VI) Manuscript writing: All authors; (VII) Final approval of manuscript: All authors. \\ Correspondence to: Ran Meng, MD \& PhD; Qi Yang, MD \& PhD. Xuanwu Hospital, Capital Medical University, Beijing 100053 , China. \\ Email: Ranmeng2011@pku.org.cn; yangyangqiqi@gmail.com.
}

Background: Distinguishing moyamoya disease (MMD) from intracranial atherosclerotic stenosis (IAS) is critical for its treatment and outcome evaluation. This study aimed to use the combined sequences of highresolution magnetic resonance imaging (HRMRI) and arterial spin labeling MR (ASL-MR) to identify the two entities accurately.

Methods: This prospective study enrolled 58 patients with middle cerebral artery (MCA) steno-occlusion identified by digital subtraction angiography (DSA), including 27 cases of MMD and 31 cases of IAS. All patients underwent MRA, HRMRI and ASL-MR prior to DSA. Two radiologists blinded to DSA results analyzed the MR images. The inner and outer diameters of the target arteries, the wall thickness of the stenotic segment, and the perfusion status in the territories of the target arteries [cerebral blood flow (CBF), cerebral blood volume (CBV) and arterial transit time (ATT)] were measured quantitatively. The differences between MMD and IAS regarding the aspects of HRMRI and Pseudo-continuous ASLMR (PCASL-MR) maps were analyzed based on both visual characteristics and data information.

Results: Regarding the HRMRI images, MMD tended to have homogeneous and concentric vesselwall thickening as well as collaterals adjacent to the stenotic vessels; while IAS showed eccentric and heterogeneous vessel-wall thickening. For the CBF maps of PCASL-MR, abnormal hyper-perfused spots embedded inside the hypo-perfused regions were observed in MMD instead of IAS. Quantitative analysis revealed that MMD displayed smaller inner and outer diameters, and smaller maximum wall thickness, higher average value of CBF, CBV and ATT, and higher maximum value of CBF and CBV, when compared to IAS (all $\mathrm{P}<0.01$ ). The average wall thickness and the maximum value of ATT showed no significant difference between MMD and IAS $(\mathrm{P}>0.01)$.

Conclusions: HRMRI combined with PCASL-MR may help distinguish MMD and IAS induced cerebral arterial stenosis and cerebral perfusion disorder accurately and non-invasively.

Keywords: Moyamoya disease (MMD); intracranial atherosclerotic stenosis (IAS); high-resolution MRI; arterial spin labeling MRI

Submitted Oct 24, 2019. Accepted for publication Dec 17, 2019.

doi: 10.21037/atm.2019.12.140

View this article at: http://dx.doi.org/10.21037/atm.2019.12.140 


\section{Introduction}

Moyamoya disease (MMD) is a rare cerebrovascular disorder characterized by the progressive stenosis of the supraclinoid internal carotid arteries and their branches, in association with a network of basal collaterals (1-5). The prevalence of MMD in East Asian was approximate 4-10 per 100,000 and an increasing trend was observed.

As a special subtype of cerebral angiopathy, MMD often inflicts both children and adults, resulting in either ischemic or hemorrhagic stroke, which differs from that induced by intracranial atherosclerotic stenosis (IAS) regarding the aspects of both treatment and outcomes $(2,6,7)$. Therefore, distinguishing MMD from IAS is essential in making a proper treatment strategy decision and assessing clinical outcomes, especially among adults.

Recent progress of neuroimaging techniques provides more precise methods to distinguish MMD from other subtypes of cerebral angiopathy. Conventional imaging examinations for detecting MMD at present include digital subtraction angiography (DSA) and magnetic resonance angiography (MRA) in combination with magnetic resonance imaging (MRI). Radiographic findings supporting the diagnosis of MMD are as follows: (I) stenosis or occlusion occurs at the terminal of the internal carotid artery (ICA) or the proximal segment of the middle cerebral artery (MCA); (II) collateral hyperplasia adjacent to the stenotic segment; (III) two or more macroscopic vascular flow voids, which are considered as hyperplastic collaterals in the basal ganglia area of the stenotic side (2).

However, sometimes even with these aforementioned imaging tools, it is still difficult to distinguish whether the arterial stenosis is originated from early stages of MMD or IAS. Moreover, DSA is an invasive method, which is not applicable in certain populations, such as those with: iodine allergies, thyroid diseases and severe renal dysfunction. Hence, this study intended to explore a new approach to distinguish MMD from IAS by using the non-invasive method: high-resolution magnetic resonance imaging (HRMRI). Meanwhile, we attempted to investigate the perfusion characteristics between MMD and IAS via the sequence of Pseudo-continuous arterial spin labeling magnetic resonance (PCASL-MR).

HRMRI is generally defined as 3-Tesla MR with the magnetic field focused on the arterial wall. In this study, we also used the $3 \mathrm{D}$ variable flip angle turbo, which is capable of proving black-blood effects to acquire clear images of the vessel wall $(8,9)$. The main pathological features of the morbid arteries in MMD include the fibrocellular thickening of the tunica intima, the thinness of the tunica media and the thinness or disruption of the internal elastic lamina (10). However, there are discrepancies between IAS and MMD regarding the pathological features of the stenotic vessel wall. HRMRI may provide a non-invasive approach to specifically distinguish the pathological changes of the stenotic segment in MMD from that of IAS.

It is well known that both MMD and IAS can result in cerebral perfusion disorders. Nonetheless, variations also exist between the perfusion disorders caused by the two entities. Pseudo-continuous arterial spin labeling magnetic resonance (PCASL-MR) is a novel, exogenous-contrastfree imaging technique used for evaluating cerebral perfusion. Instead of exogenous tracer, PCASL-MR utilizes magnetically labeled protons in the arterial water as endogenous tracer, and perfusion maps are subsequently obtained via subtraction of labeled and control signals $(11,12)$. ASL-MR has been studied for estimating hypoperfusion in both MMD and IAS $(13,14)$. However, no PCASL-MR research has been designed to explore the perfusion differences between MMD and IAS so far. In this study, we compared the features of arterial vessel-wall and the differences of cerebral perfusion between MMD and IAS prior to begin the registered study (NCT03821181).

\section{Methods}

Informed consent was obtained prior to any study-specific procedures, and this study was approved by the Institutional Ethical Committee of CCMU (Xuanwu Hospital, Capital Medical University).

\section{Patient's selection}

This study prospectively enrolled 58 patients with MCA steno-occlusion from November 2016 through November 2018 in Xuanwu Hospital. Among of them, 27 patients were confirmed as MMD by DSA, as they met the diagnostic criteria of MMD formulated by the Japanese Research Committee (15), the 31 remaining patients were diagnosed as IAS by DSA. For all patients, MRA, HRMRI as well as PCASL-MR had been performed before they underwent DSA.

\section{Inclusion criteria}

(I) Unilateral or bilateral MCA stenosis. 

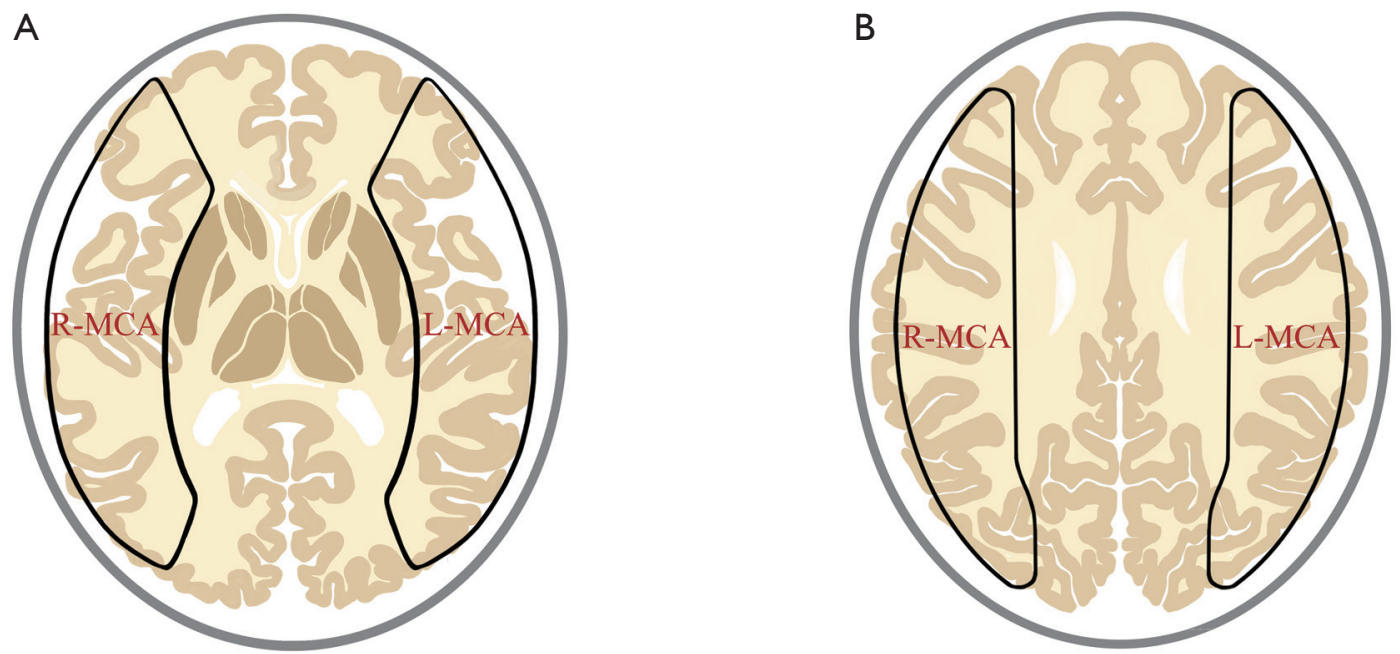

Figure 1 The images are models of middle cerebral artery perfusion territories on two brain slices (A: basal ganglia, B: semi-oval center).

(II) The degree of the shrinking of the inner diameter of stenosis $\mathrm{MCA} \geq 50 \%$ of on DSA maps or $\geq 70 \%$ on CTA/MRA maps compared to the normal inner diameter of MCA (severe stenosis of MCA on DSA/ CTA/MRA maps) (15).

(III) Stable vital signs.

(IV) No bleeding tendency.

(V) No acute ischemic stroke within 1 week.

\section{Exclusion criteria}

(I) Any ischemic (with a large infarction lesion, $\geq 1 / 3$ MCA territory) or hemorrhagic stroke within 1 week.

(II) Severe or unstable concomitant diseases.

(III) Contraindications for MR scan, such as metal implants and claustrophobia.

(IV) Acute myocardial infarction.

(V) MCA steno-occlusion secondary to other clear etiologies, such as intracranial granulomatous arteritis, and any known vasculitic disease.

\section{HRMRI scanning protocol}

All HRMRI was performed on a 3.0-Tesla system (Verio, Siemens Healthcare, Erlangen, Germany). All images were obtained based on the following parameters: acquisition time $=8-10$ minutes, echo time $=15 \mathrm{~ms}$, repetition time $=900 \mathrm{~ms}, 240 \mathrm{scan}$ slices, slice thickness $=0.53 \mathrm{~mm}$, and image-matrix $=320 \times 320$ pixels.

\section{PCASL-MR scanning protocol}

PCASL-MR was performed on a 3.0-T imaging system (Verio, Siemens Healthcare, Erlangen, Germany) with a 32-channel head coil. A multi-delay PCASL image was obtained by using an acquisition protocol with acquisition time of 5 minutes and 40 seconds, post-labeling delay of $1,500 / 2,000 / 2,500 / 3,000 \mathrm{~ms}$, echo time of $22.67 \mathrm{~ms}, 26$ scan slices, slice thickness of $5 \mathrm{~mm}$ and a $64 \times 64$ matrix resolution.

\section{Image processing and analyzing}

Data of PCASL-MR in DICOM form obtained from patients with MMD or IAS were converted to perfusion maps; the parameters including cerebral blood flow (CBF), cerebral blood volume (CBV) and arterial transit time (ATT) in the MCA territories, were processed by commercial software OsiriX (University of California, Los Angeles, America), and expressed as data from a set of region of interest (ROI) (Figure 1).

Data of HRMRI and MRA in DICOM form were observed and measured by Radiant DICOM Viewer (Medixant Company, Poznan, Poland). The outer and inner diameters as well as the arterial wall thickness in the most severe stenotic segment were measured four times with different angles using the Radiant DICOM Viewer.

Two experienced neuroradiologists who were blinded to any clinical information and DSA results evaluated all images of the patients in this study objectively, primarily 
Table 1 Baseline characteristics of all patients

\begin{tabular}{lccc}
\hline Characteristics & $\begin{array}{c}\text { MMD group } \\
(\mathrm{N}=27)\end{array}$ & $\begin{array}{c}\text { IAS group } \\
(\mathrm{N}=31)\end{array}$ & $\begin{array}{c}\text { P value } \\
\text { (independent- } \\
\text { sample } \text {-test) }\end{array}$ \\
\hline Age (y) & $\begin{array}{c}32.8 \text { (range } \\
4 \text { to 64) }\end{array}$ & $\begin{array}{c}55.9 \text { (range } \\
31 \text { to 85) }\end{array}$ & $<0.01$ \\
Male (\%) & 33.33 & 70.97 & $<0.01$ \\
Hypertension (\%) & 0 & 70.97 & $<0.01$ \\
Diabetes (\%) & 0 & 51.61 & $<0.01$ \\
Hyperlipidemia (\%) & 0 & 77.42 & $<0.01$ \\
Smoking (\%) & 0 & 29.03 & $<0.01$ \\
\hline
\end{tabular}

MMD, moyamoya disease; IAS, intracranial atherosclerotic stenosis.

focusing on the following parameters: (I) unilateral or bilateral arterial stenosis; (II) collateral vessels adjacent to the stenotic segment; (III) the status of arterial wall at the stenotic segment; (IV) abnormal vessels around the cerebral ventricle; (V) hypo- or hyper-perfusion in the stenotic MCA territories in CBF maps.

After eliminating unqualified images (MCA evanishment on HRMRI or perfusion maps with low image quality), a total of 40 CBF maps from PCASL and 40 images of HRMRI were collected for final analysis.

\section{Statistical analysis}

SPSS 21.0 (SPSS Inc., IL, USA) was used in this study for data analyses. The independent $t$-test was performed to compare the differences of outer and inner diameters, wall thickness, CBF, CBV and ATT between MMD and IAS patients. The mean and $95 \%$ confidence interval were also calculated. Two-tailed $\mathrm{P}$ value $<0.01$ was considered to indicate statistical significance.

\section{Results}

\section{Baseline characteristics of patients}

There were 18 females $(66.67 \%)$ and 9 males $(33.33 \%)$ in the MMD group, while 9 females (29.03\%) and 22 males $(70.97 \%)$ in the IAS group (independent-sample $t$-test, $\mathrm{P}<0.01)$. The median age was 32.8 [4-64] years in the MMD group and 55.9 [31-85] years in the IAS group (independent-sample $t$-test, $\mathrm{P}<0.01)$. Details are displayed in Table 1.

\section{Imaging features between MMD and IAS}

\section{HRMRI and MRA}

The enhancements of the affected arterial walls were observed in 19 patients (95\%) in the IAS and in 18 patients (90\%) in the MMD group. Concentric enhancements of the stenotic vessel walls were mainly observed in MMD rather than in IAS patients ( $85 \%$ vs. $5 \%$ ), while eccentric reconstructions were remarkable in the IAS group, Figure 2. Moreover, both the inner and outer diameters of the afflicted arteries were significantly smaller in the MMD group when compared with IAS group (MMD vs. IAS: $0.708 \pm 0.281$ vs. $1.166 \pm 0.398 \mathrm{~mm}$ of the inner diameter; and $2.984 \pm 0.515$ vs. $3.593 \pm 0.626 \mathrm{~mm}$ of the outer diameter, $\mathrm{P}<0.01)$. The artery wall thickness of the stenotic segments between MMD and IAS showed no statistical significance ( $\mathrm{P}>0.05)$. Details are shown in Table 2.

Regarding the HRMRI maps, a hazy network of small collateral vessels was seen in 19 MMD patients (95\%), among which, 11 patients (55\%) were accompanied with abnormal collateral vessels adjacent to the lateral ventricles. Nonetheless, all of these findings were not observed in patients with IAS, Figure 3.

For the MRA maps in patients with MMD, 9 (45\%) had unilateral MCA stenosis and 11 (55\%) had bilateral MCA stenosis. Meanwhile, in the IAS group, 18 patients (90\%) showed unilateral MCA stenosis and the remaining 2 patients (10\%) displayed bilateral MCA stenosis. The puffy collateral vessels around the stenotic segments were observed in 12 MMD patients (60\%), but not in the IAS group.

\section{PCASL-MR}

During visual observation, we noticed that all CBF maps in the two groups showed remarkable hypo-perfusion in the territories of the stenotic arteries. Notably, abnormal focal hyper-perfused spots embedded inside the hypo-perfused regions were found in 14 out of $20 \mathrm{MMD}$ patients (70\%); whereas, no similar finding occurred in the IAS group (Figure 3). The Suzuki stages of the 14 MMD patients (70\%) with focal hyper-perfused spots ranged from stage II to $\mathrm{V}$, and 11 of them were accompanied with abnormal collateral vessels adjacent to the lateral ventricles. The remaining $6 \mathrm{MMD}$ patients $(30 \%)$ without focal hyper-perfused spots were in stage-I and stage-VI, and no obvious moyamoya collaterals were found.

There was statistic difference between MMD and IAS regarding the following perfusion parameters: average value 
A

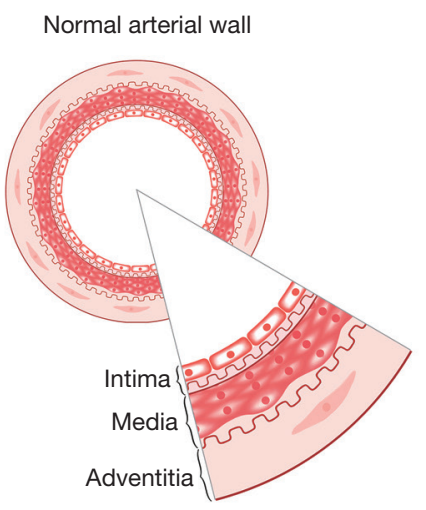

D

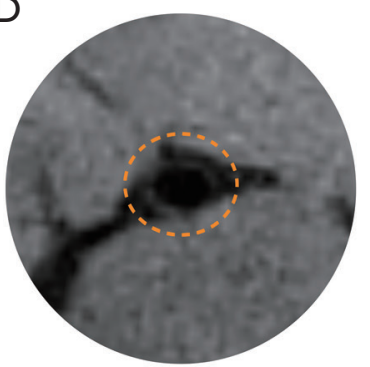

B

Moyamoya disease

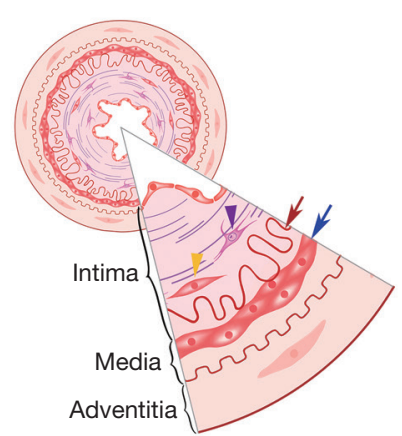

E

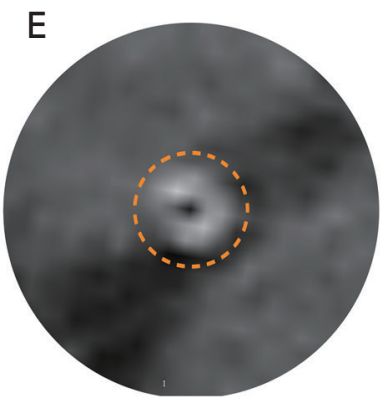

C

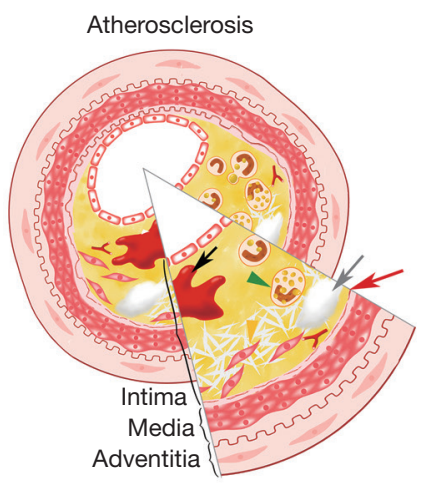

$\mathrm{F}$

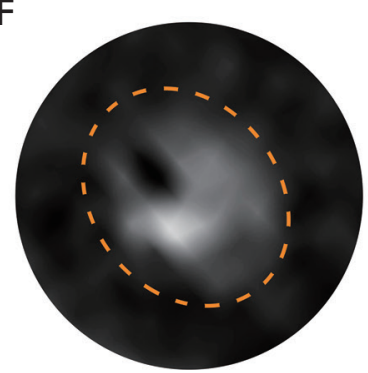

Figure $2(A, D)$ Showed the simplified image and the actual HRMRI image of the normal artery wall. (B) represented the affected artery wall in MMD, which characterized by the thickness of intima and the atrophy of media (the yellow triangle indicated the shifted and proliferated smooth muscle cells, the purple triangle indicated the fibroblast hyperplasia, the red arrow indicated the extremely tortuosed internal elastic membrane, the blue arrow indicated the decreasing of smooth muscle cells in the shrinking media). (C) Was the simplified pattern image of atherosclerosis artery wall which included the lipid deposition under the endothelium, the foam cell formation (green triangle), smooth muscle cells shifting and proliferation (yellow triangle), internal elastic membrane rupture (red arrow), calcium core (gray arrow) and internal bleeding (black arrow). (E,F) Showed the actual HRMRI image of patients with MMD or atherosclerosis. HRMRI, high-resolution magnetic resonance imaging; MMD, moyamoya disease.

of $\mathrm{CBF}, \mathrm{CBV}$, ATT and higher maximum of $\mathrm{CBF}, \mathrm{CBV}$ in the ROI of bilateral MCA territories $(\mathrm{P}<0.01)$. However, the maximum ATT value revealed no difference between MMD and IAS groups $(\mathrm{P}>0.05)$. The average value and $95 \%$ CI between the two groups are provided in Table 2 . The differences of artery wall parameters and perfusion data among different stages in MMD were illustrated in Table 3.

\section{Discussion}

Epidemiologically, the ages of onset for MMD have two peaks, including the first decade of life and age of 20-30 s, and the male-to-female ratio is approximately $1: 2$ (16). However, clinically, adult MMD-related ischemic strokes can also occur at the age of 40-70 years old. Hence, it is of great importance to distinguish between secondary vascular stenosis due to IAS and MMD, which may not be accomplished preciously by conventional imaging tools such as CTA and MRA. In this study, we intended to compare the radiographic characteristics of MMD and IAS associated vascular stenosis on the basis of imaging of HRMRI and PCASL-MR.

\section{HRMRI can distinguish the vessel-wall differences between MMD and IAS associated stenosis}

The clinical stages for MMD are often classified according to the Suzuki staging system: stage-I: narrowing at the 
Table 2 Morphological and perfusion data in the MMD group and IAS group

\begin{tabular}{lccc}
\hline & & Group & \\
\cline { 2 - 4 } Parameters & $\begin{array}{c}\text { IAS group }(\mathrm{N}=20) \\
\text { (average value and 95\% Cl) }\end{array}$ & $\begin{array}{c}\text { MMD group (N=20) } \\
\text { (average value and 95\% Cl) }\end{array}$ & $\begin{array}{c}\text { P value } \\
\text { (independent-sample } t \text {-test) }\end{array}$ \\
\hline Outer diameter $(\mathrm{mm})$ & $3.59(3.22-3.86)$ & $2.89(2.68-3.11)$ & $<0.01$ \\
Inner diameter $(\mathrm{mm})$ & $1.17(1.00-1.36)$ & $0.71(0.56-0.83)$ & $<0.01$ \\
Wall thickness $(\mathrm{mm})$ & $1.39(1.18-1.61)$ & $1.21(1.07-1.37)$ & 0.303 \\
Maximum wall thickness $(\mathrm{mm})$ & $2.10(1.77-2.36)$ & $1.50(1.36-1.65)$ & $<0.01$ \\
Mean-CBF $(\mathrm{mL} / 100 \mathrm{~g} / \mathrm{min})$ & $30.21(28.47-32.08)$ & $36.38(33.21-40.12)$ & $<0.01$ \\
Mean-CBV $(\mathrm{mL} / 100 \mathrm{~g})$ & $47.99(45.09-51.52)$ & $58.06(53.29-65.05)$ & $<0.01$ \\
Mean-ATT $(\mathrm{s})$ & $76.36(71.90-81.55)$ & $79.78(77.38-82.24)$ & $<0.01$ \\
Max-CBF $(\mathrm{mL} / 100 \mathrm{~g} / \mathrm{min})$ & $66.25(57.21-83.36)$ & $90.00(70.01-111.99)$ & $<0.01$ \\
Max-CBV $(\mathrm{mL} / 100 \mathrm{~g} / \mathrm{min})$ & $114.10(104.78-129.14)$ & $150.80(126.69-172.44)$ & $<0.01$ \\
Max-ATT $(\mathrm{s})$ & $118.75(111.52-129.32)$ & $121.95(114.34-130.95)$ & 0.033 \\
\hline
\end{tabular}

MMD, moyamoya disease; IAS, intracranial atherosclerotic stenosis; CBF, cerebral blood flow; CBV, cerebral blood volume; ATT, arterial transit time.
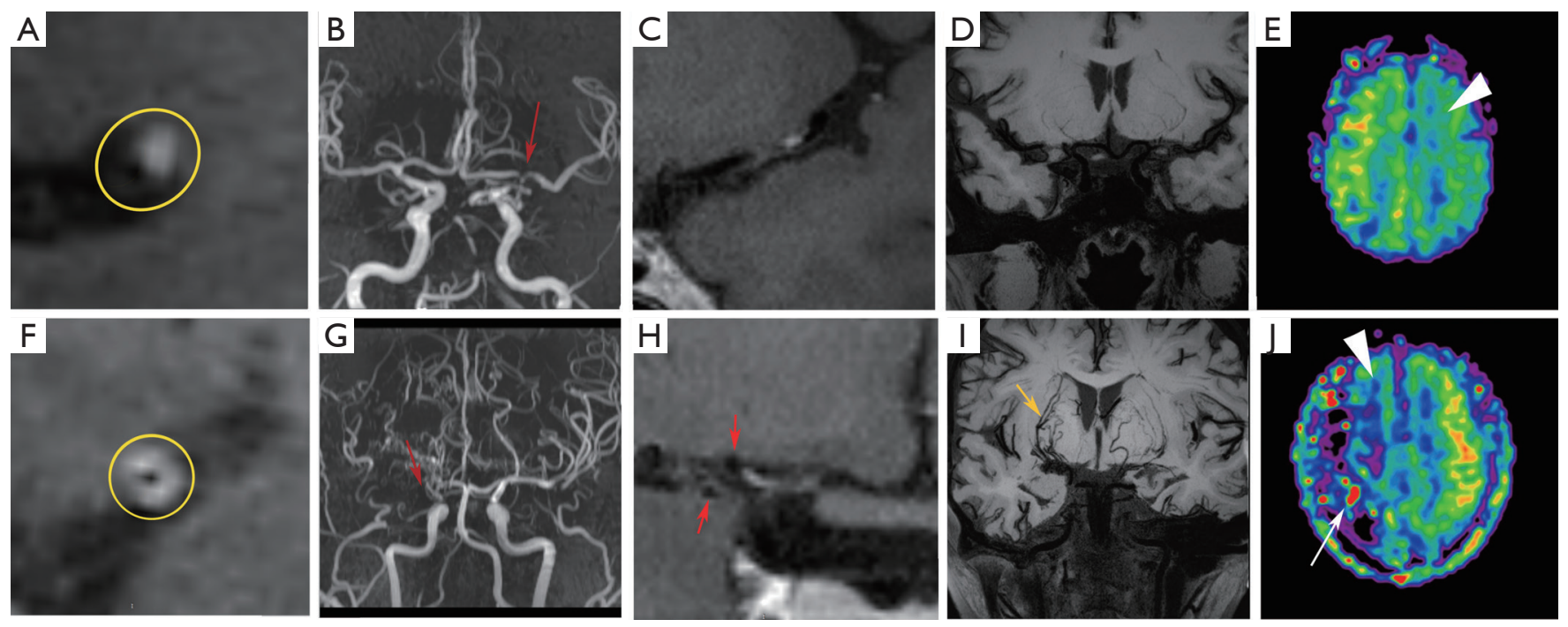

Figure 3 (A,C,D,F,H,I) Images of HRMRI. (B,G) Images of MRA. (E,J) CBF maps from PCASL. (A-E) Images from a MMD patient. (FJ) Images from an IAS patient. (A,F) The cross section of the stenotic segment (yellow circle). (B,G) The stenotic MCA. (C,H) The portrait profile of the stenotic artery (the red arrow indicated the abnormal vessel flowing void adjacent the stenosis in MMD). (D,I) Abnormal vessel adjacent to the cerebral ventricle in MMD (yellow arrow). (E,J) Hypo-perfusion areas in the stenotic MCA supply territories in the CBF maps (white triangle) and the focal hyper-perfused spots embedded inside the hypo-perfused regions (white arrow). HRMRI, highresolution magnetic resonance imaging; MRA, magnetic resonance angiography; MMD, moyamoya disease; MCA, middle cerebral artery; CBF, cerebral blood flow. 
Table 3 The figures of morphological and perfusion data in different stages in MMD

\begin{tabular}{|c|c|c|c|c|c|c|}
\hline Parameters (average) & \multicolumn{6}{|c|}{ Stages of MMD } \\
\hline Outer diameter (mm) & - & 2.734 & 2.844 & 3.573 & 2.908 & 3.006 \\
\hline Inner diameter (mm) & - & 0.618 & 0.636 & 0.964 & 0.871 & 0.738 \\
\hline Wall thickness $(\mathrm{mm})$ & - & 1.243 & 1.427 & 1.933 & 1.383 & 1.45 \\
\hline Mean-CBF (mL/100 g/min) & - & 39.837 & 34.15 & 36.676 & 26.595 & 36.26 \\
\hline Mean-CBV (mL/100 g) & - & 62.057 & 54.296 & 59.763 & 42.308 & 59.428 \\
\hline Mean-ATT (s) & - & 78.068 & 79.656 & 83.918 & 82.676 & 83.406 \\
\hline Max-CBF (mL/100 g/min) & - & 88.25 & 101.222 & 113.375 & 100 & 109 \\
\hline
\end{tabular}

MMD, moyamoya disease; CBF, cerebral blood flow; CBV, cerebral blood volume; ATT, arterial transit time.

fork of the carotid artery; stage-II: initiation of moyamoya collaterals; stage-III: aggravation of moyamoya collaterals around the narrowed vessels; stage-IV: exacerbation of the narrowed vessels and reduced moyamoya collaterals; stage-V: large vessels occlusion and more obvious reduction of surrounding moyamoya changes; stage-VI: disappearance of moyamoya collaterals, instead, the territories of the internal carotid artery system are supplied from the external carotid artery (17).

As stenotic changes only occur at the fork of the carotid artery in the absence of pronounced moyamoya collaterals at early stages of MMD, it remains a challenge to distinguish between secondary vascular stenosis due to IAS and MMD. Recently, HRMRI has been gaining an increasing attention from clinical researchers. Pathologically, there were remarkable differences between MMD and IAS associated vascular stenosis. In MMD, histopathological features in the stenotic or occlusive artery include the following: degeneration and the resultant death of arterial smooth muscle cells, causing thinning of the media; tortuous and fragmented elastic laminae, accumulation of necrotic cell components, and proliferation of arterial smooth muscle cells, causing thickening of the intima and luminal narrowing. Furthermore, in contrast to IAS, there was no significant inflammatory cell infiltration in the stenotic artery in MMD (18-20). In IAS, the main etiology of arterial stenosis is the build-up of atherosclerotic plaque, which is primarily formed with fibrous cap, foam cells, cholesterol crystal, and calcium (21). Previous studies using HRMRI have revealed features of the vascular wall of the stenotic segment in MMD: homogeneous signal intensity, concentric thickening, irregular narrowed lumen and significantly reduced outer diameter. Nonetheless, the stenotic pattern of IAS is prone to present with more eccentric and heterogeneous wall thickening, regular crescent-shaped lumen and remarkably enlarged vessel outer diameter with irregular shape $(22,23)$. Similar imaging characteristics were also observed in all enrolled 40 patients with a coincidence rate of $100 \%$.

\section{PCASL-MR may help identify the perfusion differences between MMD and IAS associated stenosis}

One of the main features of MMD includes the abnormal moyamoya collateral, which usually initiates at stage-II and remains until stage-IV.

In this study, we noticed that all CBF maps in the two groups were presented as remarkable hypo-perfusion in the territories of the stenotic arteries. Notably, abnormal focal hyper-perfused spots embedded inside the hypoperfused regions were found in $70 \%(14 / 20)$ patients in the MMD group; whereas, we did not observe similar findings in patients with IAS. We assume that the hyperperfused spots may be associated with moyamoya collateral compensation for reduced perfusion, even though sometimes these collaterals can be hardly found in MRA. 
Another explanation is that the hyper-perfused spots may be secondary to the microaneurysms on the collateral vessels $(24,25)$. Given that, this imaging characteristic may have the potential to become a useful marker for differentiating MMD from IAS.

Taken together, HRMRI combined with PCASL-MR may help distinguish causes of arterial stenosis (secondary to MMD or IAS) non-invasively and accurately. Compared to MRA and CTA, a combinatory use of HRMRI and PCASL-MR enables more precise assessment of vascular wall and inner lumen as well as cerebral perfusion status. Additionally, the total scanning time of MRA, HRMRI and ASL only takes 35-45 minutes, which is tolerable for most patients. The economic analysis of the two kinds of examination shows that DSA costs 10,000.00 RMB or more; whereas, HRMRI combined MR-ASL costs only 800.00 to 1,500.00 RMB. Moreover, HRMRI plus ASL can be used to monitor cerebral perfusion status dynamically.

There are several limitations in this study. First of all, the sample size is relatively small and all subjects were from one single center. In addition, this study did not make a hierarchical analysis of MMD for different stages. Therefore, it remains unknown whether the phenomenon of abnormal focal hyper-perfused spots embedded inside the hypo-perfused regions is present in different stages of MMD. Future work with rigorous design and larger sample size is required to further clarify these issues.

\section{Conclusions}

HRMRI combined with PCASL-MR proves to be a noninvasive, low-cost and easily accessible supplement approach of angiography, which may be helpful in the identification of etiology of cerebral arterial stenosis (MMD or IAS). Future studies providing a deeper insight into the combinatory use of the two sequences are warranted, allowing a more precise diagnosis in clinical settings.

\section{Acknowledgments}

We would like to thank all patients and doctors who participated in this study for their cooperation.

Funding: This study was sponsored by the National Key R\&D Program (2017YFC1308401), the National Natural Science Foundation (81371289) and the Project of Beijing Municipal Top Talent of Healthy Work (2014-2-015) of China.

\section{Footnote}

Conflicts of Interest: The authors have no conflicts of interest to declare.

Ethical Statement: The authors are accountable for all aspects of the work in ensuring that questions related to the accuracy or integrity of any part of the work are appropriately investigated and resolved. The study has been approved by the local ethics committees of all participating centers. All patients provided their written informed consent for data collection and analysis.

\section{References}

1. Scott RM, Edward RS. Moyamoya Disease and Moyamoya Syndrome. New Engl J Med 2009;360:1226-37.

2. Hashimoto N, Tominaga T, Miyamota S, et al. Guidelines for Diagnosis and Treatment of Moyamoya Disease (Spontaneous Occlusion ofthe Circle of Willis). Neurol Med-Chir 2012;52:245-66.

3. Kuroda S, Houkin K. Moyamoya disease: current concepts and future perspectives. Lancet Neurol 2008;7:1056-66.

4. Jeon C, Yeon JY, Jo KI, et al. Clinical Role of Microembolic Signals in Adult Moyamoya Disease With Ischemic Stroke. Stroke 2019;50:1130-35.

5. Shang S, Zhou D, Ya J, et al. Progress in moyamoya disease. Neurosurg Rev 2018;1:1-12.

6. Yamada S, Oki K, Itoh Y, et al. Effects of Surgery and Antiplatelet Therapy in Ten-Year Follow-Up from the Registry Study of Research Committee on Moyamoya Disease in Japan. J Stroke Cerebrovasc Dis2016;25:340-9.

7. Zhao Y, Zhang Q, Zhang D, et al. The Effect of Aspirin in the Postoperative Management of Adult Ischemic Moyamoya Disease. World Neurosurg 2017;105:728-31.

8. Jeffrey DB, Edward F, Richard HS, et al. High Resolution MRI: An Emerging Tool for Evaluating Intracranial Arterial Disease. Stroke 2013;1:287-92.

9. Yang Q, Duan J, Fan Z, et al. Early Detection and Quantification of Cerebral Venous Thrombosis by Magnetic Resonance Black-Blood Thrombus Imaging. Stroke 2016;47:404-9.

10. Takagi Y, Kikuta K, Nozaki K, et al. Histological Features of Middle Cerebral Arteries From Patients Treated for Moyamoya Disease. Neurol Med Chir (Tokyo) 2007;47:1-4.

11. Hartkamp NS, van Osch MJ, Kappelle J, et al. Arterial spin labeling magnetic resonance perfusion imaging in 
cerebral ischemia. Curr Opin Neurol 2014;27:42-53.

12. Hendrikse J, van Osch MJ, Rutgers DR, et al. Internal Carotid Artery Occlusion Assessed at Pulsed Arterial Spinlabeling Perfusion MR Imaging at Multiple Delay Times. Radiology 2004;233:899-904.

13. Yamamoto D, Hosoda K, Uchihashi Y, et al. Perioperative Changes in Cerebral Perfusion Territories Assessed by Arterial Spin Labeling Magnetic Resonance Imaging Are Associated with Postoperative Increases in Cerebral Blood Flow in Patients with Carotid Stenosis. World Neurosurg 2017;102:477-86.

14. Noguchi T, Kawashima M, Irie H, et al. Arterial spinlabeling MR imaging in moyamoya disease compared with SPECT imaging. Eur J Radiol 2011;80:e557-62.

15. Meng R, Asmaro K, Meng L, et al. Upper limb ischemic preconditioning prevents recurrent stroke in intracranial arterial stenosis. Neurology 2012;79:1853-61.

16. Suzuki J, Akira T. Cerebrovascular "Moyamoya" Disease Disease Showing Abnormal Net-Like Vessels in Base of Brain. Arch Neurol 1969;20:288-99.

17. Suzuki J, Kodama N. Moyamoya disease-a review. Stroke 1983;14:104-9.

18. Zhang H, Zheng L, Feng L. Epidemiology, diagnosis and treatment of moyamoya disease. Exp Ther Med

Cite this article as: Ya J, Zhou D, Ding J, Ding Y, Ji X, Yang Q, Meng R. High-resolution combined arterial spin labeling MR for identifying cerebral arterial stenosis induced by moyamoya disease or atherosclerosis. Ann Transl Med 2020;8(4):87. doi: 10.21037/atm.2019.12.140
2019;17:1977-84.

19. Lin R, Xie Z, Zhang J, et al. Clinical and Immunopathological Features of Moyamoya Disease. PLoS One 2012;7:e36386.

20. Bang OY, Fujimura M, Kim SK. The Pathophysiology of Moyamoya Disease: An Update. J Stroke 2016;18:12-20.

21. Cury RC, Houser SL, Furie KL, et al. Vulnerable Plaque Detection by 3.0 Tesla Magnetic Resonance Imaging. Invest Radiol 2006;41:112-5.

22. Kingstone LL, Shabana W, Chakraborty S, et al. Vulnerable Carotid Artery Plaque Evaluation: Detection Agreement between Advanced Ultrasound, Computed Tomography, and Magnetic Resonance Imaging: A Phantom Study. J Med Imaging Radiat Sci 2015;46:90-101.

23. Chung JW, Hwang J, Lee MJ, et al. Previous Statin Use and High-Resolution Magnetic Resonance Imaging Characteristics of Intracranial Atherosclerotic Plaque. Stroke 2016;47:1789-96.

24. Kraemer M, Keyvani K, Berlit P, et al. Histopathology of Moyamoya angiopathy in a European patient. J Neurol 2019;266:2258-62.

25. Ueno T, Sasaki T, Iwamura M, et al. Arterial Spin Labeling Imaging of a Giant Aneurysm Leading to following Cerebral Infarction. Case Rep Neurol 2018;10:66-71. 